\title{
Scientific Consensus is Dead, Long Live Scientific Consensus
}

\author{
Aaron Pital ${ }^{1}$
}

(1) Georgia Institute of Technology, Atlanta, GA; Dept. of Chemistry and Biochemistry

\section{Abstract}

NASA makes many thousands of zero-sum decisions every year regarding funding and mission planning. A primary stated goal for the outcomes of these decisions is that scientific merit be recognized and rewarded. How does one evaluate merit in a time of unprecedented publication volume? In the lack of reproducible consensus for metrics of scientific value it is human nature to fall back on heuristics that amplify bias. This same difficulty in assimilating information deforms all researchers' ability to sharpen experimental narratives through collaborative review and engagement. This whitepaper uses summary results from the 981 most-cited articles from the journal Astrobiology and 807 of their authors' complete publication histories to illustrate the accelerating problem of forging consensus in the information age, and argues that current metrics-based structures are guaranteed to result in biased outcomes. Three solutions are proposed that NASA should consider to mitigate bias, speed democratization in science, and better inform exploration in the lab and beyond.

\section{The knowledge cliff}

The central paradigm for science-based decision making is that experts know enough about conflicting data and model results to arrive at an opinion

"...the main problem [is] gauging by what context to evaluate new science at a time when research production has outpaced our traditional ways of disseminating information and generating consensus. If we are to be a community, we must have a common understanding of each other's work and interests." with biases minimized and options weighed. There exists an unspoken assumption: an expert in a given field will be de facto knowledgeable enough to fit individual claims and proposals for future work into the broader constellation of prior work and opinion. In other words, to evaluate a hypothesis you must have sufficient knowledge of alternative approaches and sufficient experience to guide the gauging of which alternatives apply in this context. This requires a working knowledge of the state of science in a field, and the amount of knowledge for broad fields can quickly eclipse human ability to assimilate information. This is the 'knowledge cliff'; when the learning curve is (humanly) untenable to remain informed regarding research within the radius of a given discipline. 
Three strategies to mitigate this difficulty in assigning merit have been 1.) to increase the number and specificity of experts involved, 2.) modify the scoring system in order to adjust for deleterious outcomes such as highlighting submissions from underrepresented groups, and 3.) falling back on heuristics of prestige such as institutional or group affiliation, educational pedigree, citation count, and past awards. NASA and other scientific bodies have implemented each of these to one degree or another depending on the context of the science questions being evaluated. The effect of these implementations varies and is beyond the scope of the claims here but suffice it to say that evidence points toward a perpetuation of systemic bias unchanged by the last 20 years of tepid promises and diversity statements.

This continued inefficacy is because none of these mitigation strategies address the main problem of gauging what context to evaluate new science at a time when research production has outpaced our traditional ways of disseminating information and generating consensus. If we are to be a community, we must have a common understanding of each other's work and interests.
What is scientific merit?

It is worth mentioning that the idea of 'objectively' assigning merit has well-evidenced issues of bias that are not covered here. The framing of the argument in this paper is that even if the traditional ways of choosing important work and assigning merit worked as advertised, there would still be unique issues due to the volume and diversity of research today.

A small thought experiment tangential to the discussion in the remainder of this whitepaper: If only median-scoring proposals had been funded by NASA for the last decade, how would science be different today? Would it be 'worse'? In what ways, and how would you know?

It's tempting to assume that researchers can narrow their focus and limit the number of publications and results they need to keep up-to-date, but for broad fields like Planetary Science and Astrobiology, how do you know when results from very different fields have bearing on your own work? How many researchers in the world have published work on sediment transport by flowing water with bearing on Mars missions? Which geochemists have studied abiotic, aqueous alteration that have bearing on organic end fate in chondrules, and what percentage are cited in NASA-funded work on the topic? How many glaciologists have insights to the behavior on Europa's surface but have research networks that do not include NASA?

If you wanted to develop a broad understanding of an interdisciplinary field like Astrobiology, a good starting place might be to read the most-cited papers of the field and their corresponding authors. That should be doable, right?

\section{How hard would it be to keep up to date?}

The 981 most-cited articles from the journal Astrobiology and full publication histories for 807 of those articles' authors, comprising some 170,000 unique articles in total were analyzed. Some selected results are shown here to illustrate the volume of knowledge piling up in our digital archives. 


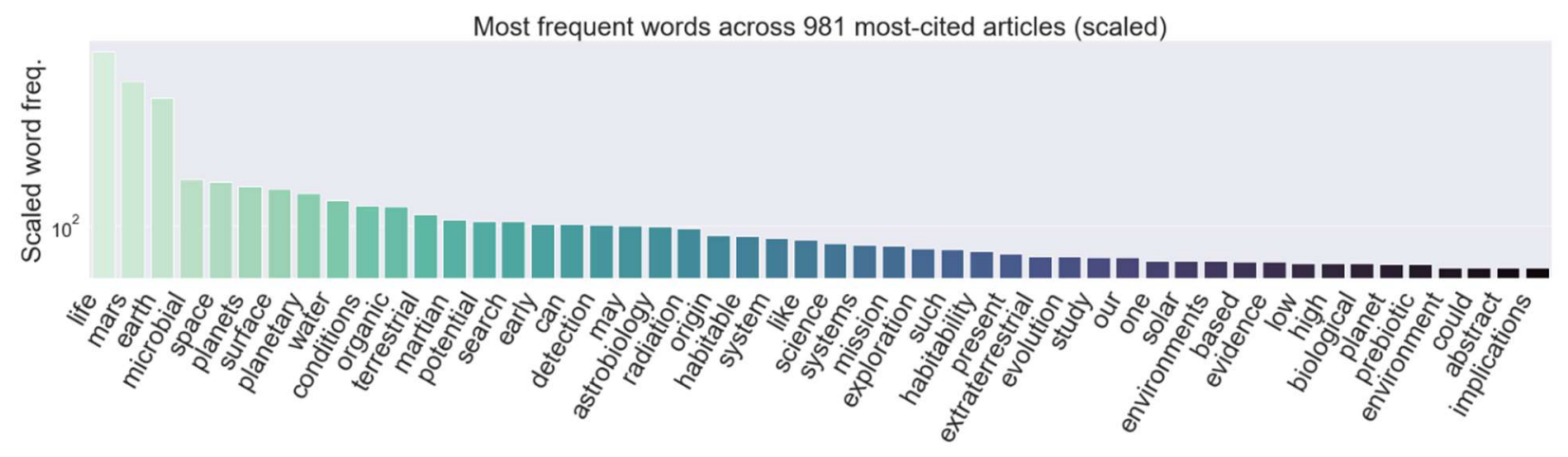

The 50 most common words from the most-cited titles and abstracts from the journal 'Astrobiology'.

If you were to spend 10 minutes reading each of the articles and do so for 8 hours a day, it would take you 20 weeks to work through the stack. These articles exist within the broader context of the field, of course, and so you might try and glean some understanding of the authors' other work and perhaps their professional affiliations. If you did this, and spent 1 second reading only the unique titles of each of their works (ignoring abstracts), this would take you an additional week working 8 hours a day.

This would likely be the only time you read many of those titles, as citation counts suggest most publications are rarely cited and presumably rarely shared or returned from search queries. All authors' citation counts follow a Pareto distribution in which a few papers are cited highly, and many papers are cited rarely. This is commonly referred to as the $80 / 20$ rule, where the top $20 \%$ of an author's publication history papers will account for $\sim 80 \%$ of their total number of citations.

The result is that most of an author's publication record is unlikely to have been read as it gets buried in searches that sort by 'popularity' metrics like citation counts or number of

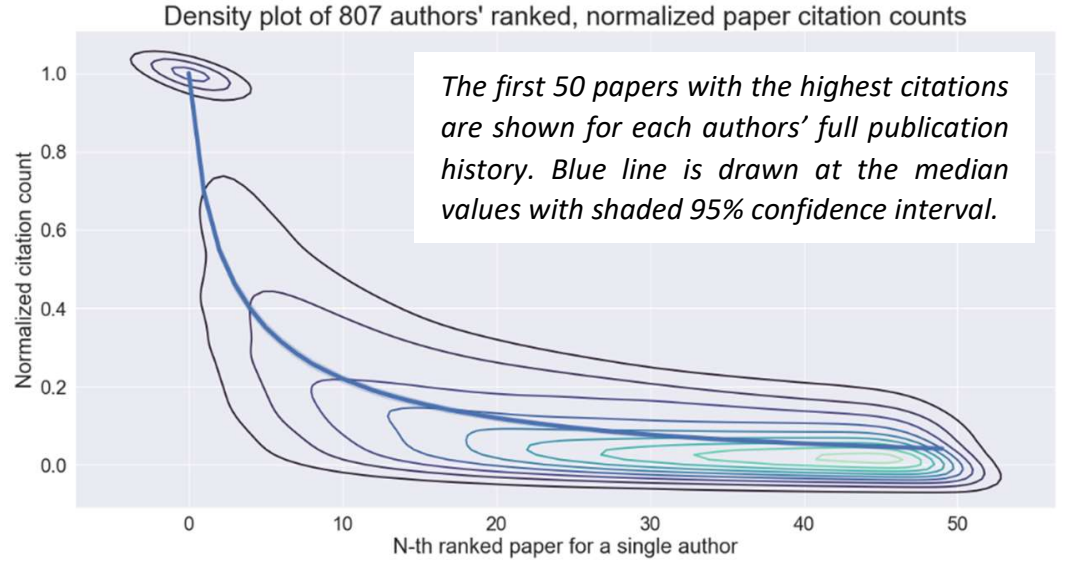

Density plot of normalized citation count by year, $2000-2020$

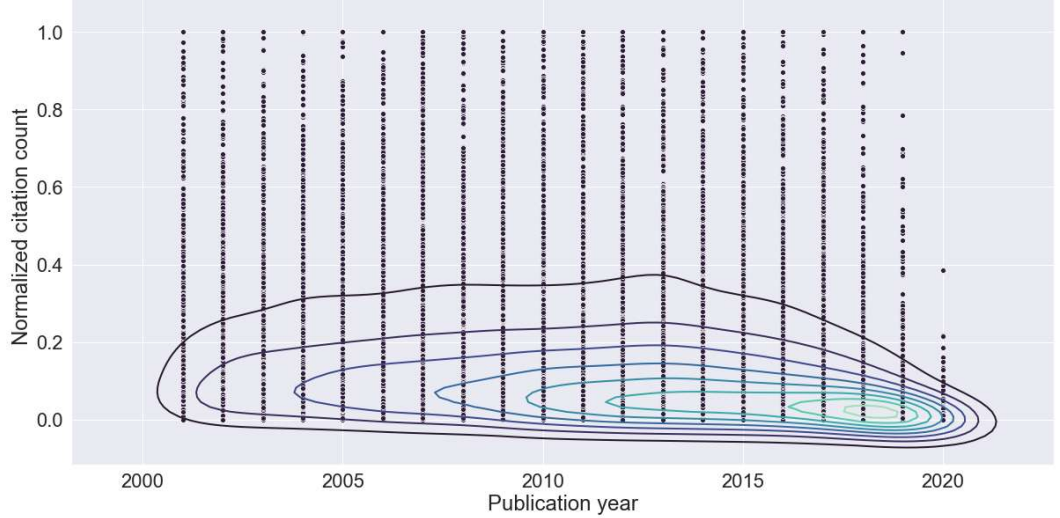

Most authors have one or two most-cited publications and many more that are sparsely or never cited. Citations increase over time, of course, but for most publications this trend is slight aggregated across all publication histories. clicks. The same algorithms that deform our social media also impact what scientific publications 
show up in search results or are recommended based on historical trends. This results in most papers being 'lost' to ether over the course of time.

Not shown here is a semantic analysis of 1.) the most-cited articles from Astrobiology, 2.) of each author's publication history, and 3.) between author's publication histories. By 'semantic analysis' I mean the word choices that act as proxies for the subject being discussed, a topic well beyond the scope of this whitepaper. This analysis shows that while general subject matter is similar across the most-cited papers (1), those authors (2) publication histories tend to be much more diverse and include a truly dizzying array of specializations from microbiology, to biophysics, to non-linear dynamic simulations.

The upshot of all of this being: if you wanted to stay up to date with an interdisciplinary field like Astrobiology (or Planetary Science) it would take an extraordinary effort to do so by directly reading publications. What is needed is an outside system to hold all these roiling interests in a single framework that can be referenced, cited, and used to understand the topology of what has yet to be known.

\section{Mapping a new consensus}

Three recommendations are proposed to enrich the Astrobiology, Planetary Science, and associated sub-communities. All three, particularly (1) and (3), also defray risks for researchers and private enterprises entering new ventures by highlighting more accurately where innovation is needed.
"Waiting for submissions and involvement from researchers disservices NASA's science readiness by incentivizing a panelproposal feedback loop of insular networks of familiar colleagues."

1.) NASA should work to map all researchers, their publication history, and areas of interest/expertise globally for fields related to Planetary Science and Astrobiology and maintain this research map as a curated, publicly accessible resource.

Several whitepapers for this decadal Planetary Science and Astrobiology survey deal with databasing and infrastructure as a means of sharing and disseminating information. This was also a minor them in the previous decadal survey. NASAs involvement with the astrophysics data system (ADS) exemplifies the feasibility of this type of project from a data standpoint and serves as a pre-existing framework to build on. Simply having the data alone is not sufficient, however, as issues of 'never-cited' and 'rarely-seen' examples in the previous section illustrate. Active involvement in soliciting feedback from applicable researchers and frequent community engagement are vital as discussed in (2) and (3) below. 
2.) NASA should use its research map to reach out to researchers pursuing science within the purview of NASA investigations in Planetary Science or Astrobiology regardless of previous interest expressed in those fields, encourage existing affiliates to collaborate with new members and each other, and encourage direct submissions from new researchers targeted through the research map.

Waiting for submissions and involvement from researchers disservices NASA's science readiness by incentivizing a panel-proposal feedback loop of insular networks of familiarity. What are the opinions outside of those seeking and receiving NASA funding? How do dogmas shape NASA's culture, and which are shared by the wider community? Funding structures have been a focus of discussion here, but the primacy of 'networking' in gaining prestige and other soft compensation by means of academic rent seeking (in the economics sense) deforms our community's sense of itself and its interests as well.

\section{3.) Regional, early career positions should be funded as a bridging or postdoctoral fellowship to liaison between local research groups, private enterprise, and NASA, fleshing out the research map (1) with active research areas and connecting individuals with funding opportunities and potential collaborators (2).}

Many aspects of research are bound up in the human experience of those who do it and do not translate easily to 'Methods' sections. NASA should seek to buttress its understanding of the state of science in the United States by funding positions that are embedded in the communities they serve and who can curate the research map with their experience. These liaisons also serve as access points (2) for NASA outreach. If NASA wants (and it should) to generate a new generation of researchers with a broad understanding of on-the-ground realities of research in interdisciplinary fields, it will have to carve out a place for them. It will also need to make a space (1) for their contributions to act as bridges that are durable.

\section{Concluding discussion}

Science has always existed at a crossroads of competing natures. The first path is the egalitarian conception of the scientific method that we're all swaddled with at grade school science fairs, when we're told that experimentation, rigor, and competence

"Most science being published today is not being viewed and that harms our ability as scientists to arrive at an informed consensus. We end up instead with a consensus of the familiar, the flashy, and the famous." decide who gets first place. The second, wider path is paved with human nature, and is the one your classmate John follows to $1^{\text {st }}$ place in the science fair with his homebuilt laser rangefinder that John's electrical engineer father paid for and helped him build.

We as a scientific community and NASA as a public resource must decide whether we're truly interested in the project of a pluralist, democratic science. The traditional consensus of scientific merit as measured by recognition, both in terms of funding and attention, will keep us from truly 
seeing each other and an ever-increasing rate over the next decade. We must choose to embrace a view of scientific consensus that knits together a community from the feudalist, insular fiefdoms we're used to.

The response that I anticipate is: "We do this already! We always try and keep an open mind. We are always willing to collaborate and accept that other researchers have a different opinion than ours." To this I say that it is possible to have good intentions with no intention of doing good. Most science being published today is not being viewed and that harms our ability as scientists to arrive at an informed consensus. We end up instead with a consensus of the familiar, the flashy, and the famous. We end up with a body scientific that is numb to the largest portion of itself. In a time of unprecedented tools for managing data and connecting with each other we have an opportunity to chart a new path forward, and NASA can lead the way.

\section{Further Reading and General References}

Kay, W.D., 2012. Defining NASA: the historical debate over the agency's mission. SUNY Press.

Launius, R., 2012. Imprisoned in a Tesseract: NASA's Human Spaceflight Effort and the Prestige Trap. Astropolitics, 10(2), pp.152-175.

McNamee, S.J. and Miller, R.K., 2009. The meritocracy myth. Rowman \& Littlefield.

Sousa, R., 2008. Research funding: less should be more. Science, 322(5906), pp.1324-1325.

Voosen, P., 2017. NASA pushes for diversity in planetary science. 